\title{
Part Per Billion
}

National Cancer Institute

\section{Source}

National Cancer Institute. Part Per Billion. NCI Thesaurus. Code C70565.

A unit of proportion equal to $10 \mathrm{E}-9$. 